\title{
British Academy of Cosmetic Dentistry
}

\section{$\mathrm{T}$} he British Academy of Cosmetic Dentistry (BACD) was formed in November 2003 by a group of dentists, many of them leading practitioners in the field of cosmetic dentistry. They saw a need to share their knowledge with others and offer a previously unexplored level of cosmetic dental education within the UK. The aim of creating a dynamic and active group of dentists and dental technicians remains strong, with the role of all dental team members - including dental hygienists, dental therapists, practice managers and assistants - having grown in the overall aesthetic dentistry experience. This is equally important to the Academy's ethos.

There are currently just under 700 active members of the BACD. Dental professionals can become a member by registering via the BACD website. The Academy offers different levels of membership, including:

- Level 1 - Associate Membership

- Level 2 - Full Membership

- Level 3 - Accredited Membership

- Level 4 - Fellowship

- Student Membership

- Non-Membership.

Associate Membership is available to all dental team members, with the membership rates varying depending on your job title. As an Associate member dentist or dental technician, you can apply and qualify for Full membership, which carries further benefits but no additional fees.

For a dentist or dental technician to become an Accredited member, they must satisfy all the criteria of the Academy's Accreditation Programme to prove competence in key areas of cosmetic dentistry. This is a highly-regarded level of membership, and all BACD members have the opportunity to follow this path. Fellowship of the BACD is the highest and final stage of membership, and enables Accredited members to demonstrate excellence in interdisciplinary treatment planning and the execution of complex treatments to consistently high standards.

Dental students can also benefit from BACD membership at a monthly cost of $£ 5$ or an annual fee of only $£ 40$. For those who are unsure whether they are ready to join the BACD, BACD offers a unique 'nonmembership', which entitles you to access Recommended Meetings for just $£ 199$ per session, and other BACD events at a 'nonmember' rate.

Currently, any dentist can offer cosmetic dental treatment irrespective of their training or experience beyond the basic dental degree. There are no specialist qualifications and no specialist register for cosmetic dentistry. Moreover, techniques constantly progress, and the public are becoming increasingly educated and discerning, so it is essential for dental professionals to stay up to date.

The BACD supports its members and offers opportunities for self-improvement through world class education within a collaborative environment, where dental professionals can come together to share their knowledge and experience. This is so that everyone can become better at what they do - regardless of their level of prior knowledge. Ultimately, BACD wants to make a difference to people's lives by providing exceptional, ethical cosmetic dentistry.

BACD is proud to offers its members access to a wide range of social and educational events held across the UK throughout the year - including Recommended Meetings, Ladies Who Do Dentistry, Young Dentist Day, and the highly-anticipated Annual Conference, which involves hands-on training. These events not only provide a platform for dental professionals to hone their knowledge and skills through exceptional dental education, but also enable members to network with peers and many of the industry's most distinguished professionals.

The BACD strives to help strengthen relationships within the industry, which

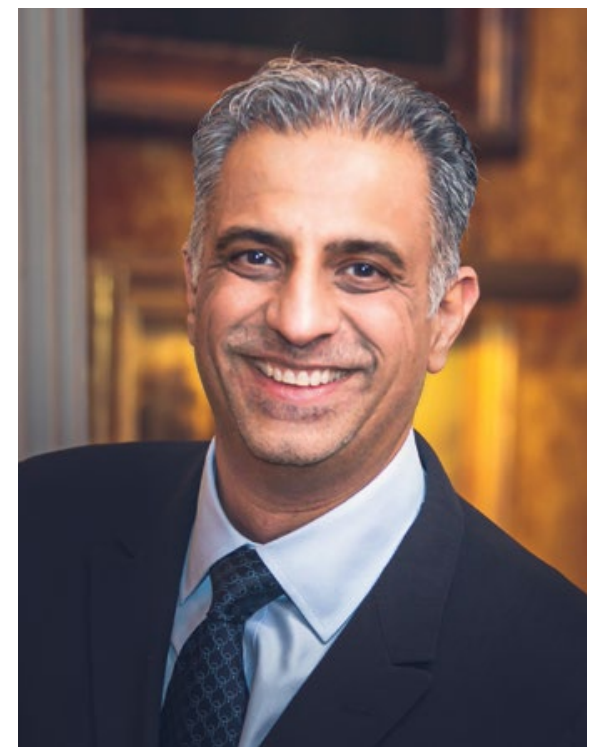

President of BACD, Nishan Dixit

is why it also provides access to the trade. Members of the BACD can explore cuttingedge dental materials and technologies from leading manufacturers, whilst taking advantage of exclusive trade partnership discounts on the latest products.

In addition, the Academy offers members a pathway for progression through its prestigious Accreditation Programme. This has been designed to enable dentists and dental technicians to demonstrate that they can diagnose, plan, and execute exceptional cosmetic dental treatment safely, ethically, and competently. Successfully completing the Accreditation Programme will earn candidates a unique accolade for their expertise in delivering the highest quality treatment, as judged by their peers and leaders from the field.

Beyond accreditation, the BACD affords mentoring and career support to help clinicians reach their full potential. Members also have access to BACD cosmetic consultation protocols and consent forms, which can be used to ensure that the best course of treatment is advised for each patient. Furthermore, BACD members benefit from being able to display the BACD logo and other branded material as a mark of cosmetic dental excellence, in order to elevate their practice.

Having become the largest cosmetic dental academy in Europe, the BACD has established itself on the world stage as one of the most influential and forward-thinking 
difganisations in the profession. It continues to raise awareness and promote the need for dental professionals to update their knowledge and skills, and expand on their experience to deliver the best possible treatment for patients. Our drive to progress cosmetic dentistry is a key contributor to our growth in BACD membership. As a result, we are able to offer even greater value year-on-year.

The BACD is determined to strengthen its current offerings so that members can make the most of their investment. It is keen to continue developing its social and educational programme by offering access to more events, which will provide the chance for members to learn from worldrenowned clinicians. The BACD is also looking to collaborate with other professional organisations from the industry. This is all part of BACD's goal to remain a highly inclusive and innovative community of dental professionals, who are dedicated to upholding uncompromising ethical standards with the utmost integrity and passion.

https://bacd.com/

\section{US celebrates 75 years of community water fluoridation}

In commemoration of the seventy-fifth anniversary of community water fluoridation (CWF) in the US, the eight US Public Health Service (USPHS) Chief Dental Officers from 1981 to 2018 have all signed a statement to reaffirm their support and commitment to CWF.

In 1945, the US Surgeon General and the National Institute of Dental Research sponsored the first water fluoridation implementation in Grand Rapids, Michigan, and a study of data from that effort demonstrated a $60 \%$ drop in dental caries among Grand Rapids children after just 11 years. Progress in CWF since then has resulted in fluoridation in 46 of the 50 largest cities in the country and for populations served by community water systems, over $70 \%$ benefit from having optimum levels of fluoride.
The US Centers for Disease Control and Prevention describe CWF as

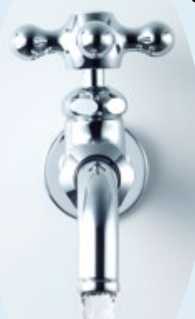

one of ten great public health achievements of the twentieth century.

In 2001, Surgeon General David Satcher, who commissioned the first-ever Surgeon General's Report on Oral Health, stated that 'more than 50 years of scientific

research has found that people living in communities with fluoridated water have healthier teeth and fewer cavities...'

The commemorative statement by the eight Chief Dental Officers, published 7 July 2020, signs off: 'Please join us in resolving to support CWF, one of the greatest public health achievements and a cornerstone to the prevention of dental caries and improvement of both oral health and overall health' 